\title{
A New Judgment of Intoxication Based on Changes in Low Frequency Characteristics of Speech Signal
}

\author{
Seong-Geon Bae ${ }^{\# 1}$, Won-Hee Lee ${ }^{* 2}$ and Myung-Jin Bae ${ }^{* * 3}$ \\ ${ }^{\# 1}$ Division of Computer Media Information Engineering, Kangnam University, Gugal-dong, Giheung-gu, \\ Yongin-si, Gyeonggi-do, Korea \\ sgbae@kangnam.ac.kr \\ ${ }^{* 2-3}$ Department of Information and Telecommunication Engineering, Soongsil University, 369 Sangdo-Ro, \\ Dongjak-Gu, Seoul, Korea \\ 2vbluelovev@ssu.ac.kr, ${ }^{3}$ mjbae@ssu.ac.kr
}

\begin{abstract}
This research examines parameters of drinking judgment by comparing sound characteristics before and after drinking using voice analysis. Until now, characteristics of drinkers are studied from perspectives of pitch, formant, and changes in size of sound, however, as these factors are very sensitive to environmental changes to be used as a measurer for judging drinking, it is important to find more accurate parameters. Especially, changes in high frequency and low frequency have to be separated to identify sound characteristics and thus, if environment changes this many lead to calculation of inaccurate parameters. Therefore, this research studies about characteristics before and after drinking by using addition signal, which enables application to various environment along with more accurate analysis.
\end{abstract}

\section{Keyword-Judgement of intoxication, High frequency signal, Formant, Pitch}

\section{INTRODUCTION}

What we cannot avoid in our social life is drinking. Sometimes, people recover relationship through drinking but it could also worsen the condition. What is more, Korean companies gather together for drinking to have a better cooperation among the staff. However, too much drinking may lead to the worsening of the relationship. That is, drinking is something that has both advantages and disadvantages. Few years ago, sobriety test has to be done on the road where cars come and go. However, recently, various tools for the test is used such as mobile application, GPS, or the car engine does not start if the driver seats down and alcohol is detected by the drinking measurer installed in the handle.

These attempts are short distance measuring methods that can be done if alcohol detectors are located close to the driver. In contrast, if sobriety is measured by sound this can be done even from a far distance. For transportations such as train, ship, airplane, and cars, there are many difficulties of checking sobriety in a close distance. Therefore, detecting sobriety through sound analysis enables detection from both short and long distance [1]. There are quite many difficulties to applying those existing analysis tools to the indirect areas. Those methods focus on changes in formant or pitch as their main judgment standards for testing alcohol level. These characteristics adopt a method of finding unique parameter by researching features before and after drinking in various ways. As sobriety test based on sound signals varies strongly depending on individual characteristics, sound signals well represent each person. However, as they are so sensitive to environmental changes and still, not perfectly fit to be used in different environment. Due to above-mentioned reasons, researches so far are done based on general signal processing system [2][3].

As this is the case, this research has identified these characteristics to carry out study on addition signals. In Chapter 2, it discusses about previous methods of analyzing parameters and followed by this, Chapter 3 is about explanation on characteristics of addition signals and frequencies. The last Chapter 4 makes final conclusion by talking about experiment used in this research and its result and future research directions.

\section{CHARACTERISTICS OF OVERLOADED SIGNAL}

The general method of analyzing sound signals is done by dividing sound characteristics into pitch and formant. This has been widely used because this processes typical features of individuals as well as common features of the sound by separating them into time scope and frequency scope. Therefore, as they analyze sound based on its creation model, it can be applied in various fields for use. Also, these analyzed sound signals are 
used in analysis and synthesis. Although calculation of frequency scope takes lot of time but as it allows accurate division of frequency factors it is considered as one of most effective methods. First of all, input sound signal goes through low pass filter of $3.75 \mathrm{kHz}$ and then, removes unnecessary factors. What is left behind after removal is composed of all the important factors for sound signal. In frequency scope, Fast Fourier Transformation (FFT) is carried out to separate the most remarkable pitch and formant. For those separated signals, their characteristics are analyzed by each frame to examine characteristics before and after drinking [2][3]. Although there is a high possibility of universal application but it' $\mathrm{d}$ be hard to measure if different environments are considered. In regards to this, this research has applied addition signals to overcome these limits.

\section{PROPOSED METHODS}

Before going through regular sound analysis which is to divide pitch and formant, this research has analyzed characteristics of addition sound. In previous researches, differential signals are used to amplify high frequency and this was very useful for analyzing naturalism and clarity [3]. Characteristics of addition signals are they have features of low frequency but at the same time, they are very useful for studying pronunciation and respiration of the sound or its system. First of all, addition sound can be calculated as shown in Fig. 2. The input sound signals generally separate unnecessary factors through cross wave in low frequency. Here, neighbor signals which have the highest correlation are added to the computation of addition signals. Those neighbor signals with high correlation play a role of emphasizing low frequency factors and reducing high frequencies. Therefore, this research has carried out study based on above characteristics.
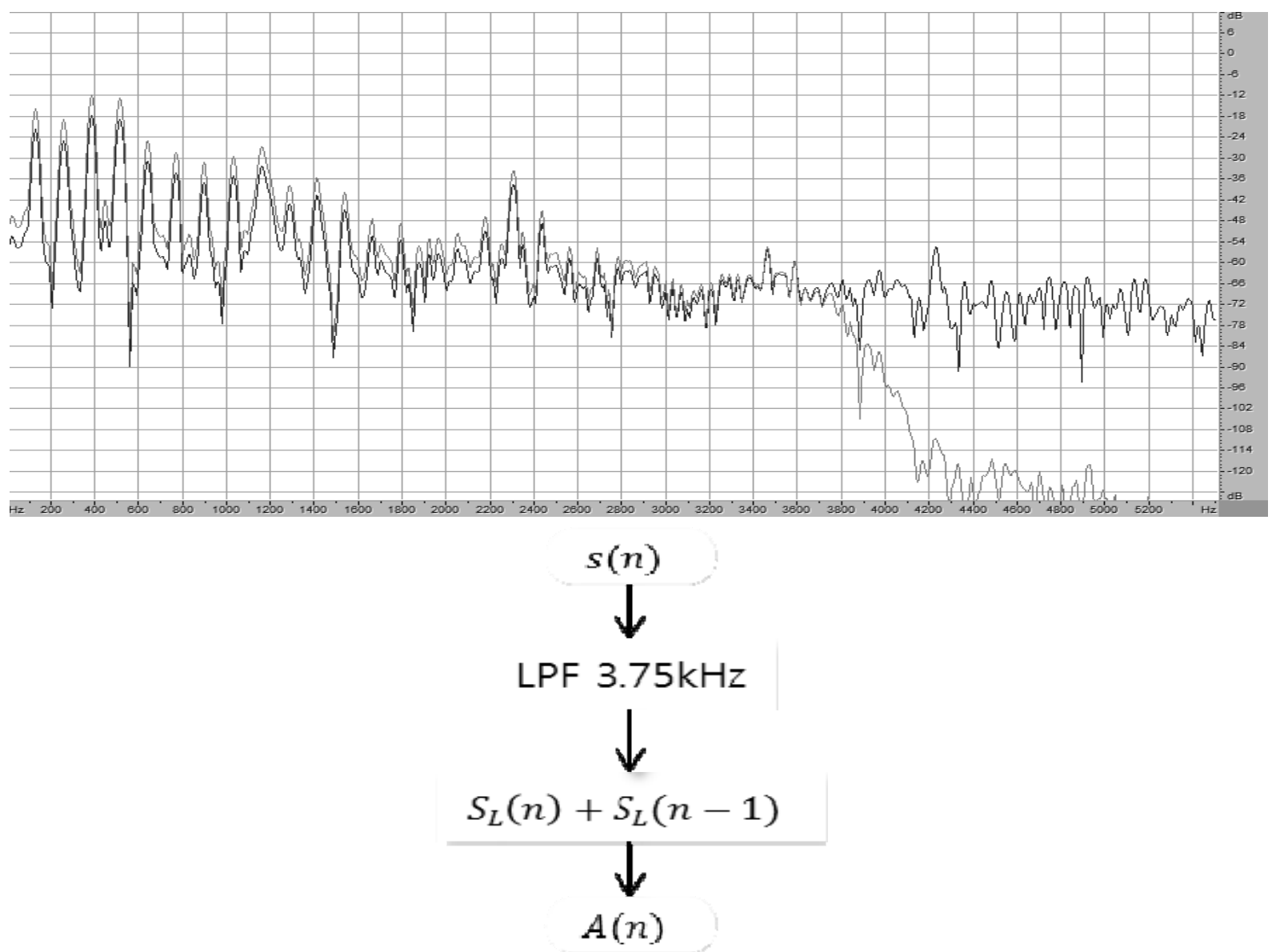

Figure 1. Proposed a flow chart

Calculated signals shown in Fig. 1, they even more emphasize low frequency factors by adding signals with the highest correlation. The reason for having such computation is to analyze environmental features by emphasizing low frequency signals. 


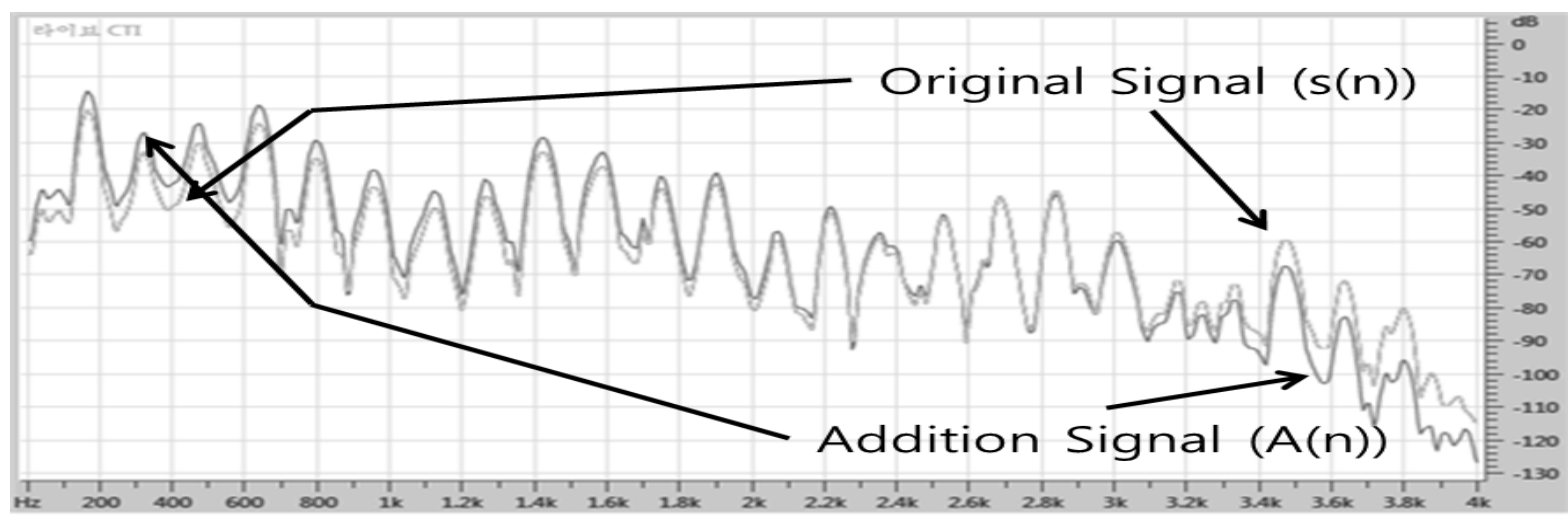

Figure 2. Compare original signal s(n) to addition signal A(n)

Fig. 2 shows a comparison of those characteristics on frequency. It is about frequency features of original and addition signals. In terms of addition signal, it tends to be emphasized in low frequency scope compared to low frequency but in high frequency scope, it is reduced. Such features can be examined by the fact that there is a big energy difference in frequency. Especially for low frequency analysis, it is possible to compare various features of those frequencies and thus, this research has also applied them into this study. Addition signals tend to emphasize low frequency but reduce high frequency. Therefore, it is very useful for analyzing environmental influence. This will play an important role in identifying pronunciation and respiration of sound or its system.
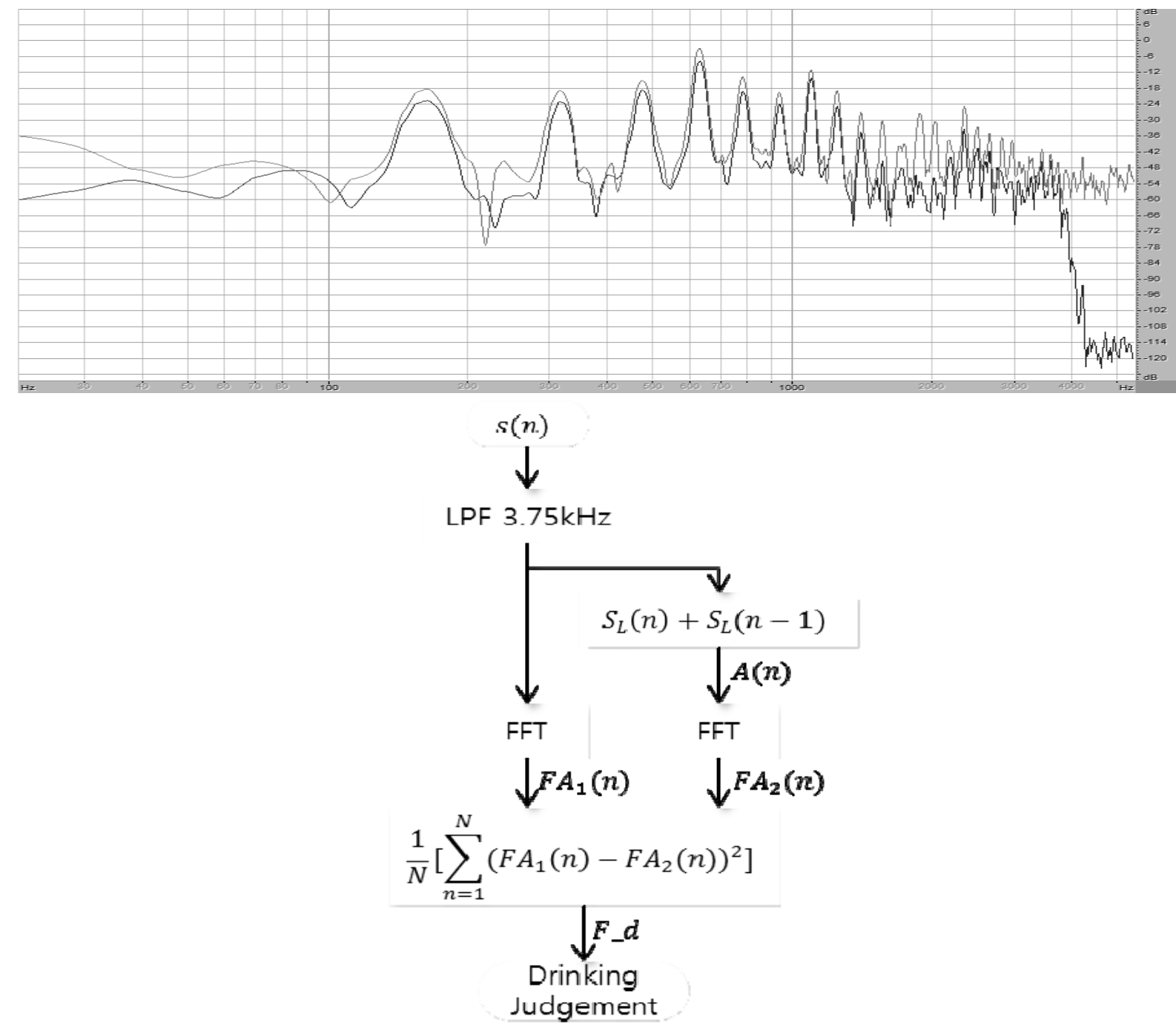

Figure 3. Proposed a flow chart for judging intoxication 
Fig. 3 is about algorithm for sobriety judgment. Here, addition signal of input sound signals are calculated. To find out characteristics of addition and original sounds they are calculated by frame. Afterwards, FFT is done for both original and addition signals. To obtain difference between frequencies in signals that went through FFT, distance between frequency factors are calculated and is applied to determining sobriety. $F A_{1}(n)$ in formulation for determining sobriety means FFT of original signals and FFT of additions signals are indicated as $F A_{2}(n)$.

\section{CONCLUSION}

Short-distance sobriety check is only possible if detecting device is located close to the person. Due to this reason, there are various problems related to this such as human rights or accidents. Therefore, ways to determine sobriety from a far distance is also required. As this is the case, this research studied parameters which identify features shown when one drinks alcohol through analysis of addition signals. Generally, analysis on pitch and formant are done to examine sound signals but in this research, it has used parameters which emphasize features of low frequency signals and reduce features of high frequency. The most important factor is that it is applicable without using database of the drunken driver. In the future, this information will be a standard for obtaining important information changes in recognition as well as addition fields and become developed into a new way of checking sobriety.

\section{REFERENCES}

[1] Won-Hee Lee, Myung-Jin Bae, "Reducing Errors of Judgment of Intoxication in Overloaded Speech Signal,” IJET Vol.8, No.1(2016), pp.219-224.

[2] Won-Hee Lee, Seong-Geon Bae and Myung-Jin Bae, "A Study on Improving the Overloaded Speech Waveform to Distinguish Alcohol Intoxication using Spectral Compensation,” IJET Vol.7, No.5(2015), pp.1957-1964.

[3] Changjoon Jung, Seonggeon Bae, Myungsook Kim and Myungjin Bae, "Speech Sobriety Test Based on Formant Energy Distribution," IJMUE Vol.8, No.6(2013), pp.209-216.

[4] Seonggeon Bae, Myungjin Bae, “A New Speech Coding using Harmonics Emphasis Filter," ISAAC 2013, AACL Vol. 1(2013), pp4344.

[5] Seonggeon Bae, Myungsook Kim, and Myungjin Bae, "On Enhancement Signal Using Non-uniform Sampling in Clipped Signals for LTE Smart Phones ”, IEEE ICCE-berlin(2013), pp.125 - 126.

[6] Seonggeon Bae, Hyungwoo Park and Myungjin Bae, "On a New Enhancement of speech Signal using Nonuniform Sampling and Post Filter,"ICHIT 2012, LNCS 7425(2012), pp.723-729, Springer-Verlag.

[7] Geumran Baek, Myungjin Bae, "Study on the Judgment of Intoxication State using Speech Analysis ". Vol.352 CCIS, 2012, pp. 277282, Springer-Verlag. 2012.

[8] Marcieli Bellé, Sílvia do Amaral Sartori, Angela Garcia Rossi. "Alcoholism: effects on the cochleo-vestibular apparatus". Rev Bras Otorrinolaringol. 73(1):116-22. 2007.

[9] Tahwinder Upile1, Fabian Sipaul1, Waseem Jerjes1, "The acute effects of alcohol on auditory thresholds, BMC Ear, Nose and Throat Disorders". 7: 4 doi : 10.1186/1472-6815-7-4. 2007.

[10] Behne, D. M., \& Rivera, S. M. (1990). Effects of alcohol on speech: Acoustic analysis of spondees. Research on Speech Perception, Progress Report, 16 (pp. 263-291). Bloomington, IN: Speech Research Laboratory.

\section{AUTHOR PROFILE}

Seong-Geon Bae. He received the Ph.D. degree in Telecommunication \& Information from Soongsil University in 2014. He is currently the Professor of the Division of Computer Media Information at Kangnam University.

Won-Hee Lee. He is currently the under Ph.D. degree in Telecommunication \& Information from Soongsil University.

Myung-Jin Bae. He received the Ph.D. degree in Electronic Engineering from Seoul National University in 1987. He is currently the Professor of the Dept. of Information \& Telecommunication at Soongsil University. He has authored and coauthored more than 300 journal articles and conference papers. His research interests are Speech Signal Processing, Audio Processing and Speech Communications. 